\title{
Simultaneous tune and coupling feedback in the Relativistic Heavy Ion Collider, and possible implications for the Large Hadron Collider commissioning
}

\author{
P. Cameron, A. DellaPenna, L. Hoff, Y. Luo, A. Marusic, C. Schultheiss, and S. Tepikian \\ Brookhaven National Laboratory, Upton, New York 11973, USA \\ M. Gasior and R. Jones \\ CERN, European Organization for Nuclear Research, CH-1211 Geneva 23, Switzerland \\ C. Y. Tan \\ Tevatron Department, Fermi National Accelerator Laboratory, Batavia, Illinois, USA
}

(Received 9 October 2006; published 14 December 2006)

\begin{abstract}
Simultaneous tune and coupling feedback were successfully implemented during RHIC run 6. In this paper we describe the measurement and control hardware and software used to accomplish this, present some of the results, discuss areas that require further investigation, and finally offer a few comments on possible implications of these results for LHC commissioning.

DOI: 10.1103/PhysRevSTAB.9.122801

PACS numbers: 29.20.Dh, 29.27.Eg, 29.27.Fh, 41.85.Qg
\end{abstract}

\section{INTRODUCTION}

The Relativistic Heavy Ion Collider (RHIC) is a versatile facility, capable of operating with polarized protons and a variety of ion species over a broad range of energies. Acceleration ramp requirements change frequently to meet the evolving needs of the physics experiments. There has been an ongoing effort at RHIC [1] to implement betatron tune feedback as a tool for ramp development, and possibly to control tune during routine operations. Early efforts $[2,3]$ were hampered by the requirement for large dynamic range, as well as the effect of large betatron coupling. Both problems have been addressed, the first by an improved analog front end [4-6], and the second by continuous measurement and correction of coupling $[7,8]$.

In hadron accelerators, to minimize emittance growth and the consequent reduction in luminosity available to the physics experiments, the beam excitation needed to reliably operate phase-locked loop (PLL) tune measurement systems must be very small. The power in the resulting betatron signal is typically of the order of femtowatts, while the power delivered to the pickup unrelated to the betatron tune is of the order of watts. In addition, transition crossing in RHIC introduces complications resulting from bunch shortening and fast orbit, tune, and chromaticity changes. Much effort was devoted to solving this problem of dynamic range, which was ultimately dealt with by the direct diode detection analog front end (3D AFE).

PLL tune tracking and feedback become impossible as coupling becomes large. Coupling rotates the planes of betatron oscillation (the eigenmodes) away from the planes in which the magnet portion of a tune feedback loop applies corrections. When this rotation exceeds 45 degrees the magnet loop applies corrections in the wrong plane and the feedback is driven unstable. To deal with this, additional data acquisition channels were added to the PLL to measure the amplitude and phase of both eigenmode projections in both planes, making possible continuous coupling measurement, and eventually feedback.

These improvements were incorporated into a new VME-based tune and coupling measurement and feedback system that was assembled for RHIC run 6. The system performed as expected "out of the box," tracking tunes and measuring coupling within minutes of the first circulating bunched beam.

The two RHIC rings are cooled to helium temperature sequentially, with about one week elapsing between the time when the first and second rings are cold. During that week, preliminary tuning of the PLL system and integration with controls and the magnet system was completed. At the end of the week, with both rings cold, ramping commenced. The first ramp was done with feedbacks off, to establish a baseline. For the next ramp feedbacks were on, and decoupled beam was delivered to full energy. To the best of our knowledge, this successful ramp was the world's first attempt at beam acceleration in a synchrotron with simultaneous tune and coupling feedback.

\section{COUPLING FORMALISM}

A brief description of the coupling formalism [9] is presented here. Figure 1 shows the eigenmodes and their projections. In the most general case the eigenmodes are ellipses, which are not orthogonal to each other, as shown in the right portion of the figure. The left portion shows the special case when the minor axes of the ellipses vanish. The frequency of mode 1 is denoted by $Q_{1}$, while $A_{1, x}$ and $A_{1, y}$ represent the amplitudes of this mode in the horizontal and vertical plane, respectively. Similarly $\phi_{1, x}$ and $\phi_{1, y}$ represent the phases of this mode in the horizontal and vertical plane, respectively. The same notation applies for mode 2. 

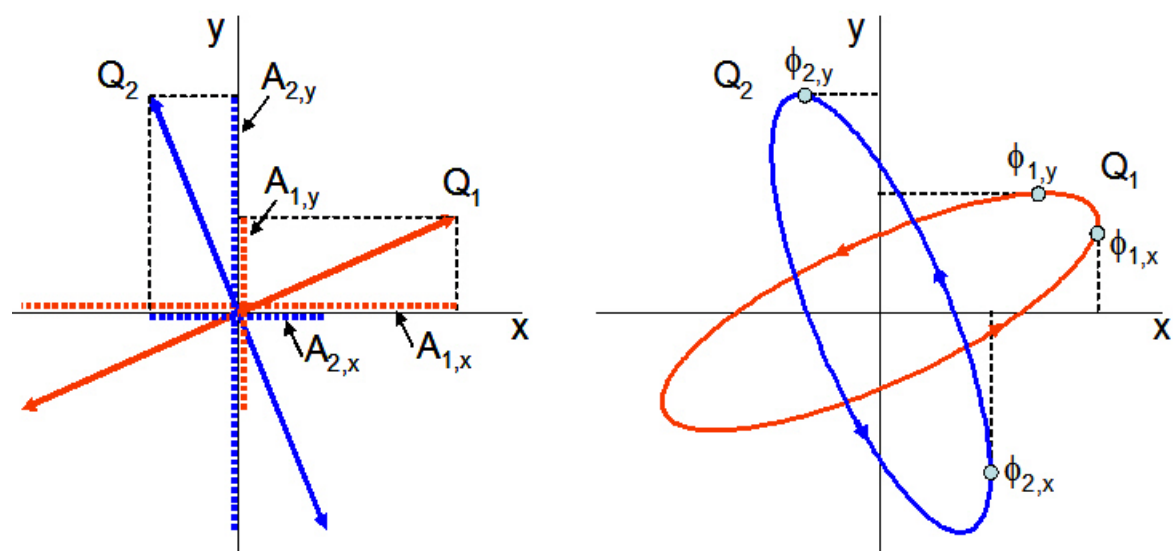

FIG. 1. (Color) Schematics showing the two eigenmodes rotated with respect to the horizontal and vertical planes due to coupling.

All of the quantities shown in the figure are measured by the PLL. From this data, the following parameters can be calculated:

$$
\begin{aligned}
r_{1} & =\frac{A_{1, y}}{A_{1, x}}=\sqrt{\frac{\beta_{y}}{\beta_{x}} \frac{\left|C^{-}\right|}{2 \nu+\Delta},} \\
r_{2} & =\frac{A_{2, x}}{A_{2, y}}=\sqrt{\frac{\beta_{x}}{\beta_{y}} \frac{\left|C^{-}\right|}{2 \nu+\Delta},} \\
d \phi_{1} & =\phi_{1, y}-\phi_{1, x}, \\
d \phi_{2} & =\phi_{2, x}-\phi_{2, y},
\end{aligned}
$$

where $\beta_{x}$ and $\beta_{y}$ are the horizontal and vertical beta functions at the pickup, and

$$
\begin{gathered}
\nu=\frac{1}{2} \sqrt{\Delta^{2}+\left|C^{-}\right|^{2}}, \quad\left|C^{-}\right|=\frac{2 \sqrt{r_{1} r_{2}}\left|Q_{1}-Q_{2}\right|}{\left(1+r_{1} r_{2}\right)}, \\
\Delta=\frac{\left|Q_{1}-Q_{2}\right|\left(1-r_{1} r_{2}\right)}{\left(1+r_{1} r_{2}\right)} .
\end{gathered}
$$

When the eigenmodes are decoupled they are in the horizontal and vertical planes, the ratios $r_{1}$ and $r_{2}$ are equal to zero, the ellipses collapse to straight lines, and the coupling phases $d \phi_{1}$ and $d \phi_{2}$ are equal to zero. The coupling amplitude $C^{-}$is often referred to as $d Q_{\min }$, or the minimum tune split. The parameter $\Delta$ is the difference between the fractional parts of the unperturbed, or "set" tunes. When the eigenmodes are decoupled, the eigentunes (measured) and the unperturbed (set) tunes are identical. The unperturbed tunes can be calculated by

$$
\begin{aligned}
& Q_{x, 0}=Q_{1}+\frac{1}{2} \Delta-\frac{1}{2} \sqrt{\Delta^{2}+\left|C^{-}\right|^{2}}, \\
& Q_{y, 0}=Q_{2}-\frac{1}{2} \Delta+\frac{1}{2} \sqrt{\Delta^{2}+\left|C^{-}\right|^{2}} .
\end{aligned}
$$

\section{SYSTEM ARCHITECTURE AND SIGNAL PROCESSING}

Figure 2 shows a block diagram of the tune and coupling measurement and feedback system. Analog processing of

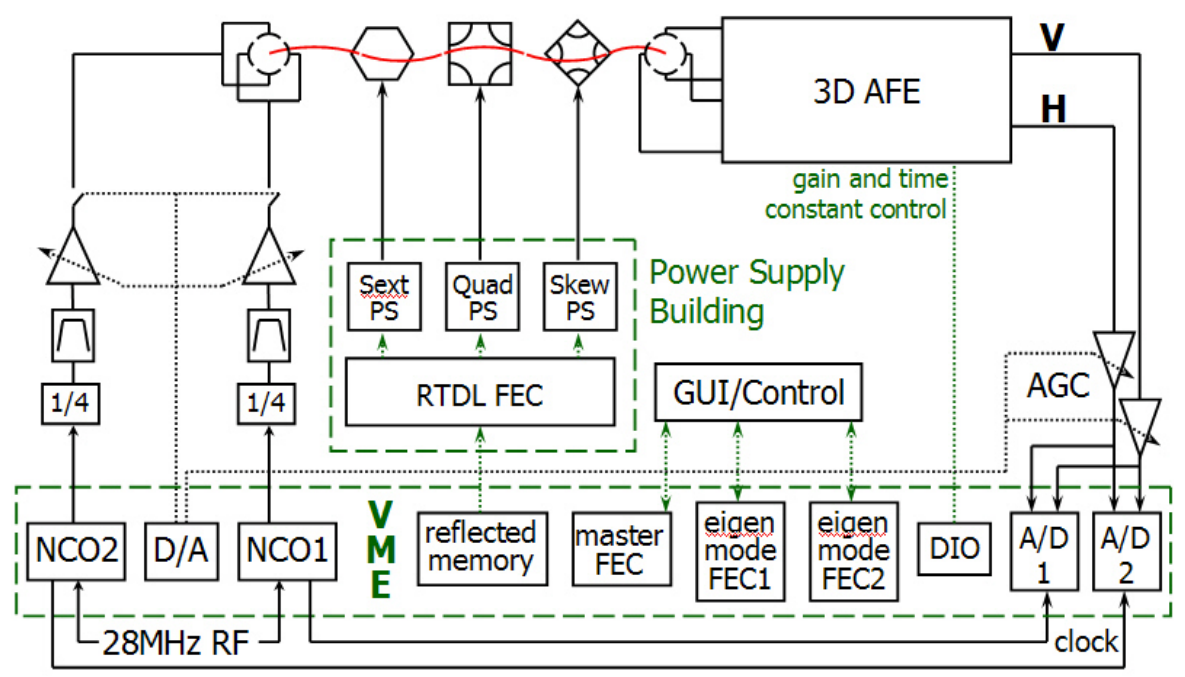

FIG. 2. (Color) System block diagram. 
signals from the pickup is accomplished by the 3D AFE. After detection, filtering, and amplification the tune signals from the two pickup planes are split and digitized. Digital processing is accomplished in two signal paths that, with the exception of coupling correction, are independent. The two signal paths provide the data needed to maintain phase lock to the two eigenmodes, and to correct coupling and tune, and eventually chromaticity.

When the eigenmode PLLs are locked, the digitizer clocks are phase synchronous to the eigenmodes. As shown in the figure, the digitizer clocks are generated by numerically controlled oscillators (NCOs), one for each eigenmode. The NCOs are beam-synchronously clocked from the $28 \mathrm{MHz}$ RF. Their output is at 4 times the betatron frequency, permitting simple I/Q demodulation.

Data for each eigenmode is processed by a front end computer (FEC) dedicated to that eigenmode. Processed data is passed to a master FEC that calculates magnet correction strengths. Corrections are written to a reflected memory module that is mirrored via dedicated fiber to a similar module in the power supply building, where a FEC applies the corrections to the power supplies. Kicker and signal path gains, as well as the 3D AFE gains and time constants, are controlled by additional VME modules. The RHIC implementation accomplishes measurement and control for both the "blue" and "yellow" rings in a single VME crate, and the number of VME modules in that crate is approximately twice that shown in the diagram.

\section{A. Kicker and pickup}

The kicker design originated at CERN, and was driven by two considerations. The first was the need to excite the rigid $7 \mathrm{TeV}$ LHC beam without excessive kicker power, and the second was to couple the kicker effectively to the beam without presenting excessive impedance to the beam when viewed in its reciprocal role as a pickup.

Figure 3 shows the relative contributions of the electric and magnetic fields to the total field in the pickup as a function of the kicker impedance, at constant power. It is clearly advantageous to favor the magnetic field, and to

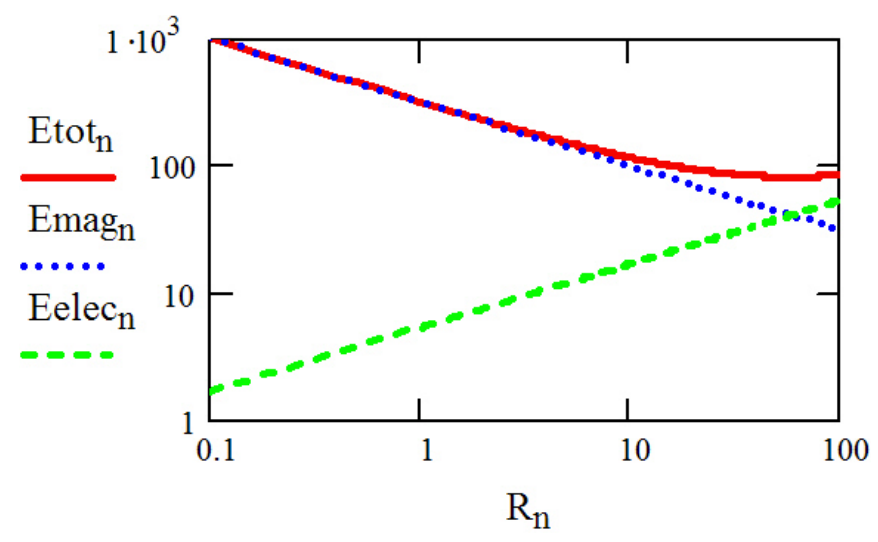

FIG. 3. (Color) Kick as a function of impedance.

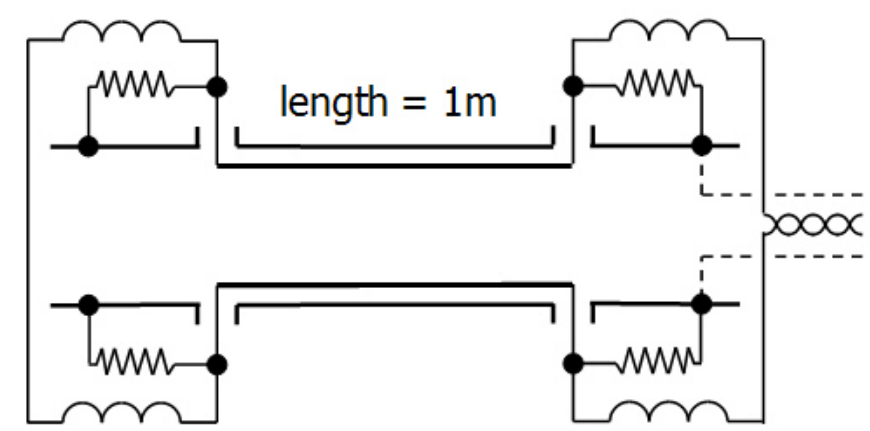

FIG. 4. Kicker block diagram.

operate at the lowest practical impedance. The PLL operates at baseband, where transmission line effects can be ignored, and the minimum practical impedance is then defined by the resistance of the cables, connectors, feedthroughs, and striplines, as well as by the current carrying capability of the feedthroughs. This approach eases the task of exciting the beam without excessive kicker power.

Figure 4 shows the kicker layout adopted for RHIC. The kicker is a $1 \mathrm{~m}$ long dual plane device. The $50 \Omega$ striplines subtend 70 degrees, and their inside diameter is $11 \mathrm{~cm}$. The design exploits the fact that kickers and pickups are not truly reciprocal devices $[10,11]$. A kicker has response at DC, but a pickup does not. The kicker is driven in series through the four $1 \mu \mathrm{H}$ inductors shown in the figure. Their impedance is $\sim 0.1 \Omega$ at the $\sim 20 \mathrm{KHz}$ betatron frequency of RHIC, but more like $400 \Omega$ at the $75 \mathrm{MHz}$ response peak of the $1 \mathrm{~m}$ stripline when viewed as a pickup. The inductors permit low impedance driving of the device as a kicker, and effectively block its response as a pickup, protecting the driver amplifier. The $50 \Omega$ resistors shown in the figure provide a matched termination for the pickup response of the striplines.

The kicker is driven differential mode through shielded twisted pairs. The system has been operated both with the 3D AFE in the tunnel, and with beam signals cabled out on the heliax. There are no problems with bleedthrough across the $\sim 160 \mathrm{~dB}$ difference between the kicker path and the signal path, as long as single point grounding is maintained with the beampipe serving as ground.

The pickup is identical in construction to the kicker. The signal is taken from the upstream ports, and the downstream ports are terminated in $50 \Omega$. Beta functions at the pickup are $\beta_{h} \sim 25 \mathrm{~m}$ and $\beta_{y} \sim 130 \mathrm{~m}$. Motion control is available for centering the pickup on the beam if so desired. Our experience has been that beam centering in the pickup is not critical, and motion feedback has not been implemented.

\section{B. Direct diode detection analog front end}

The crucial part of the 3D AFE is a peak detector. Two such detectors connected to opposing electrodes of the pickup, as shown in Fig. 5, yield the amplitude modulation 


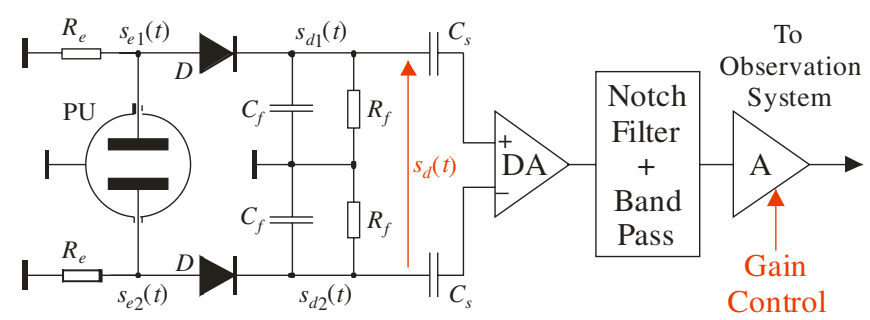

FIG. 5. (Color) Block diagram of a 3D AFE.

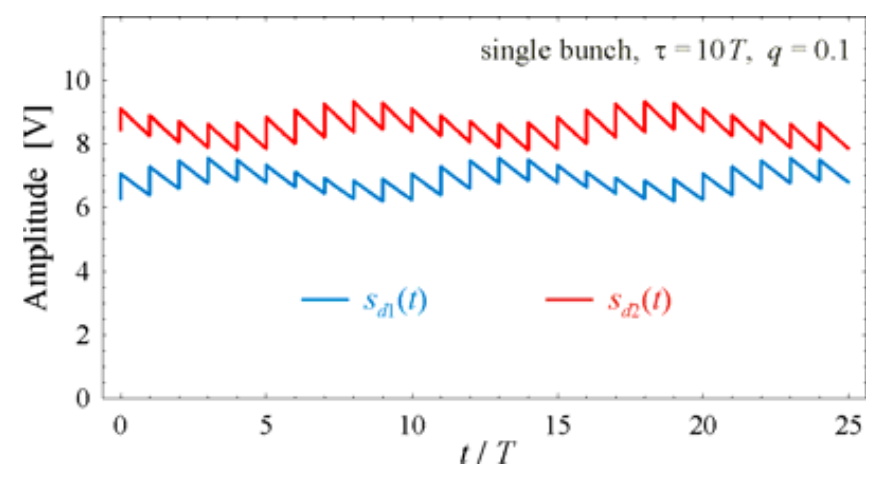

FIG. 6. (Color) Example of peak detector voltages.

envelope of the beam signals. Such signals, depicted in Fig. 6, are superposed on a DC voltage related to the bunch amplitude. The signal difference, shown in Fig. 7 for a single bunch, contains almost the whole bunch modulation amplitude, with a DC value related to the beam offset from the center of the pickup. Since the DC content can be suppressed using a series capacitor, the corresponding revolution frequency $\left(f_{r}\right)$ background can already easily be removed by some $50 \mathrm{~dB}$ before the first amplifying stage.

The 3D circuitry in Fig. 5 can be thought of as two sample-and-hold blocks, sampling bunch signals close to their maxima at the bunch repetition rate and downmixing the wideband bunch spectrum into the baseband. Note that a 3D AFE is "low frequency" only after the detectors, due to the "time stretching" of the short beam pulses. Before the detectors, the processed bandwidth can extend into the $\mathrm{GHz}$ range, covering the full coherent spectrum in hadron machines. In the detection process the spectral content from this wide bandwidth is converted to the baseband, resulting in a system sensitivity in the nanometer range for high intensity beams.

As sketched in Fig. 5, the peak detector voltages with the DC content removed by the series capacitors are subtracted by the differential amplifier (DA). This further increases the suppression of $f_{r}$ and improves the immunity of the system to interference. The filters further attenuate $f_{r}$ by some $100 \mathrm{~dB}$, as seen on the AFE characteristic in Fig. 8. The overall $f_{r}$ suppression from all system stages can be estimated to be some $160 \mathrm{~dB}$. Another advantage of the 3D

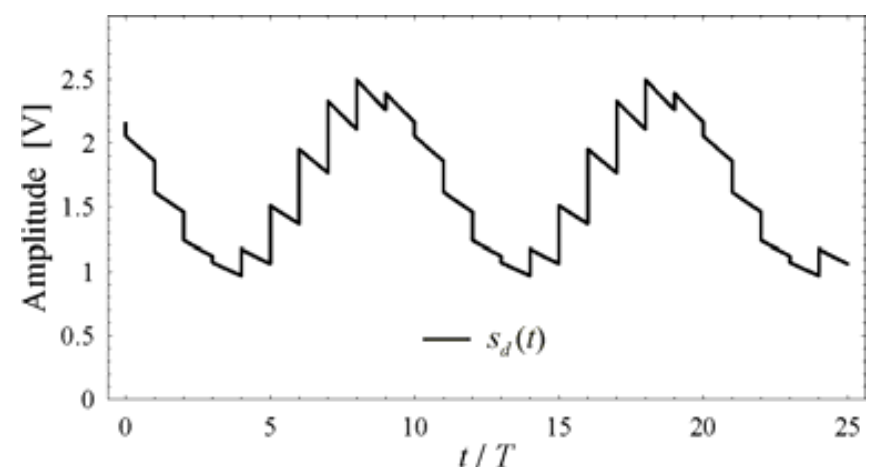

FIG. 7. Difference of the signals in Fig. 6.

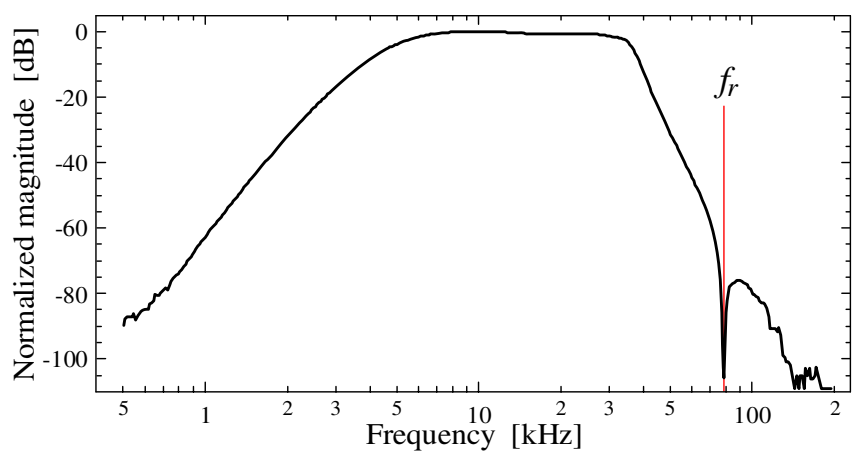

FIG. 8. (Color) RHIC AFE frequency characteristic.

approach is that the output signal, which is in the audio range for large machines, can be efficiently digitized with high resolution audio analog-to-digital converters (ADCs).

Gain in the 3D AFE is adjustable over a range of $65 \mathrm{~dB}$ in $5 \mathrm{~dB}$ steps. In addition, it is useful to have continuous gain control in both the kicker and signal paths to stabilize the overall system loop gain under varying beam conditions. Provision has been made to accomplish this by monitoring signal strength and the signal-to-noise ratio. A feedback loop can be closed in the signal path to maintain constant signal strength, and a similar loop in the kicker path to maintain constant signal to noise.

\section{PLL signal processing}

Commercial-off-the-shelf (COTS) components were widely used in assembling the data acquisition and processing systems. Two exceptions are the standard RHIC V108 "utility" module, and the numerically controlled oscillators (NCOs). The V108 module supports a variety of utility functions, such as crate temperature monitoring and decoding RHIC timing and global data signals. Its chief role in the present system is to associate RHICspecific time stamps to data. The NCO is a standard RHIC module typically used in RF applications. The important characteristics for an NCO in this application include appropriate frequency range, support for external 
clocking to automatically track RF frequency changes during beam energy ramps, and smooth transitions during frequency adjustments to maintain phase continuity.

The software executes in the standard RHIC software environment. This includes the VxWorks operating system, device drivers (typically written in the " $\mathrm{C}$ " language), and a RHIC-specific abstraction layer called the accelerator device object (ADO) layer, developed using a $\mathrm{C}++$-like language and stored in files called "rad files."

Hardware modules used in the system are shown in Table I. Device drivers were already available for all hardware modules, and ADOs were available for most of the needed functions, including analog and digital control, crate monitoring, time stamp decoding, and even tune feedback (from a previous generation RHIC system). Two new ADOs were developed for data acquisition and processing. One supports closed-loop feedback on kicker strength and/or pickup signal gain. The other supports data acquisition and processing associated with tune and coupling measurement and will be described in detail in the following paragraphs. Four of the $5 \mathrm{CPU}$ modules in the system are assigned to the four eigenmodes of the 2 RHIC rings. Each of these CPUs runs a single instance of the data acquisition ADO and two instances of the feedback ADO, one for kicker strength and one for signal gain. The fifth CPU collects tune and coupling data from the other CPUs computes tune and coupling correction, and broadcasts those values to magnet power supply controllers. The basic scheme for tune feedback had been demonstrated in a previous generation RHIC tune feedback system.

The ADC module is configured to continuously acquire data into one of two "ping-pong" buffers. The sampling clock is provided by the NCO at 4 times the betatron frequency, so that ADC samples contain alternately inphase and quadrature data. The number of samples in the buffers is configured to be a multiple of 4 to preserve the relative phase of samples within each of the two buffers. The "buffer full" interrupt initiates one pass of data processing. During this pass, the data is separated into averages of the in-phase and quadrature values, which are then low-pass digitally filtered. The resulting quadrature value is the error signal to a proportional-integral-derivative
(PID) loop which adjusts the NCO frequency, realizing a PLL locked onto the betatron frequency.

To measure coupling, the signal from the orthogonal plane is acquired via a second ADC channel, using the same sampling clock. The resulting signal is demodulated using the same technique as the primary channel.

\section{Coupling correction and magnet control}

For tune and coupling feedback in both of the RHIC rings, a total of 20 data streams (the 8 eigenmode projections for each of I and Q, plus the 4 tunes) are passed from the 4 eigenmode FECs to the master FEC, where amplitudes and phases are calculated from the I and Q data. For each projection, the projection amplitude is given by $\sqrt{I^{2}+Q^{2}}$. The projection phase is given by $a \tan 2(Q, I)$. If the eigentunes are well tracked, the measured phases $\phi_{1, x}$ and $\phi_{2, y}$ are driven to zero by the functioning of the PLL. The phase of the coupling coefficient is given by $\phi_{1, y}$. With the eigentune split and the amplitude ratios $r_{1}$ and $r_{2}$, from Eq. (2) the coupling coefficient amplitude is determined. From Eq. (3), the uncoupled set tunes are also obtained.

For coupling correction, RHIC has 3 skew quadrupole families, F1, F2, and F3. The coupling contributions from these 3 families are about 120 degrees apart when they are powered with the same strength and proper polarities. For coupling correction we combine F1 and F3 with the same strength to produce a family F13 whose contribution to the total coupling coefficient is approximately orthogonal to that only from F2 family. Knowing the coupling coefficient at the location of the PLL pickup, based on the accelerator optics model the global decoupling strengths for the two orthogonal families F2 and F13 can be calculated. In reality, the coupling angles of F2 and F13 are slightly modified with the measured phases of the eigenmode projections.

The feedback loop for tune control [12] has the measured tunes as inputs. The differences between the measured and desired tunes are the error signals, from which are calculated the focusing and defocusing quadrupole strength outputs. Implicit in the operation of the tune feed-

TABLE I. VME modules used in the tuned feedback system.

\begin{tabular}{lll}
\hline \hline Function & \multicolumn{1}{c}{ Model } & \multicolumn{1}{c}{ Notes } \\
\hline ADC & VMIVME 3123 & 16 channel, 16 bits, external clock, ping-pong buffers \\
DAC & VMIVME 4140, 4122 & 8 channel, 16 bits \\
Digital output & VMIVME 2170 & 32 channel \\
CPU & MVME2100 & 200 MHz PowerPC 603 \\
NCO & RHIC RF & External clock \\
Utility & V108 & Provides time stamps \\
Shared memory & MM-6702 & Nonvolatile SRAM w/battery backup \\
Reflective memory & VMIVME 5576 & Broadcast calculated magnet corrections \\
\hline \hline
\end{tabular}


back loop is the assumption that the tunes are decoupled, so that the measured eigentunes correspond to the correction planes of the feedback loop. The two planes typically have different frequency responses. The error signals from each plane are filtered separately, permitting the overall loop responses of the two planes to be equalized. A PID filter is utilized as a compensation filter. The magnet feedback loop for coupling control is similar to the tune feedback loop.

\section{RESULTS}

Using data from an acceleration ramp during RHIC run 5 , the coupling parameters $C^{-}$and $\mathrm{D}$ were calculated from Eq. (2), and are shown in the upper pane of Fig. 9.

The lower pane shows the measured and (calculated) set tunes, and clearly shows crossing of the set tunes during the ramp. One's understanding of the quality of tune control in Fig. 9 would be very different without the availability of
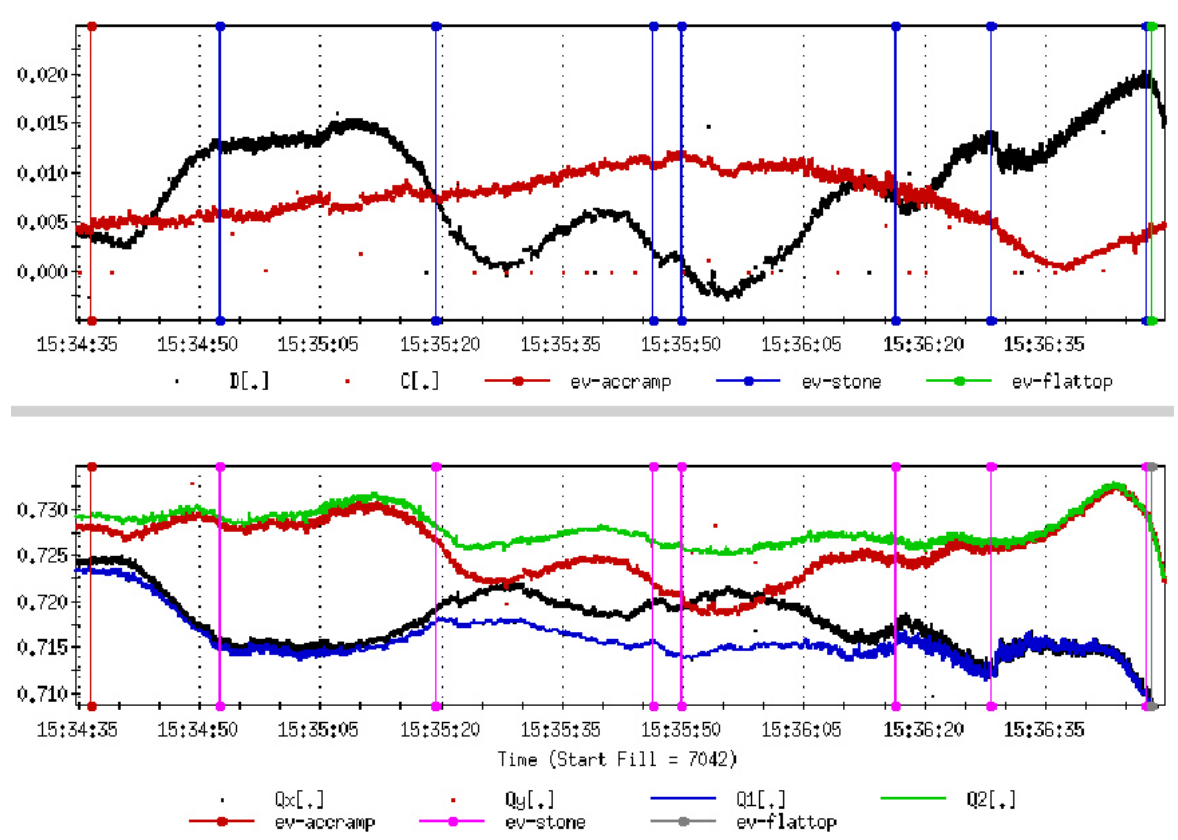

FIG. 9. (Color) Coupling parameters, measured tunes, and set tunes during a ramp.
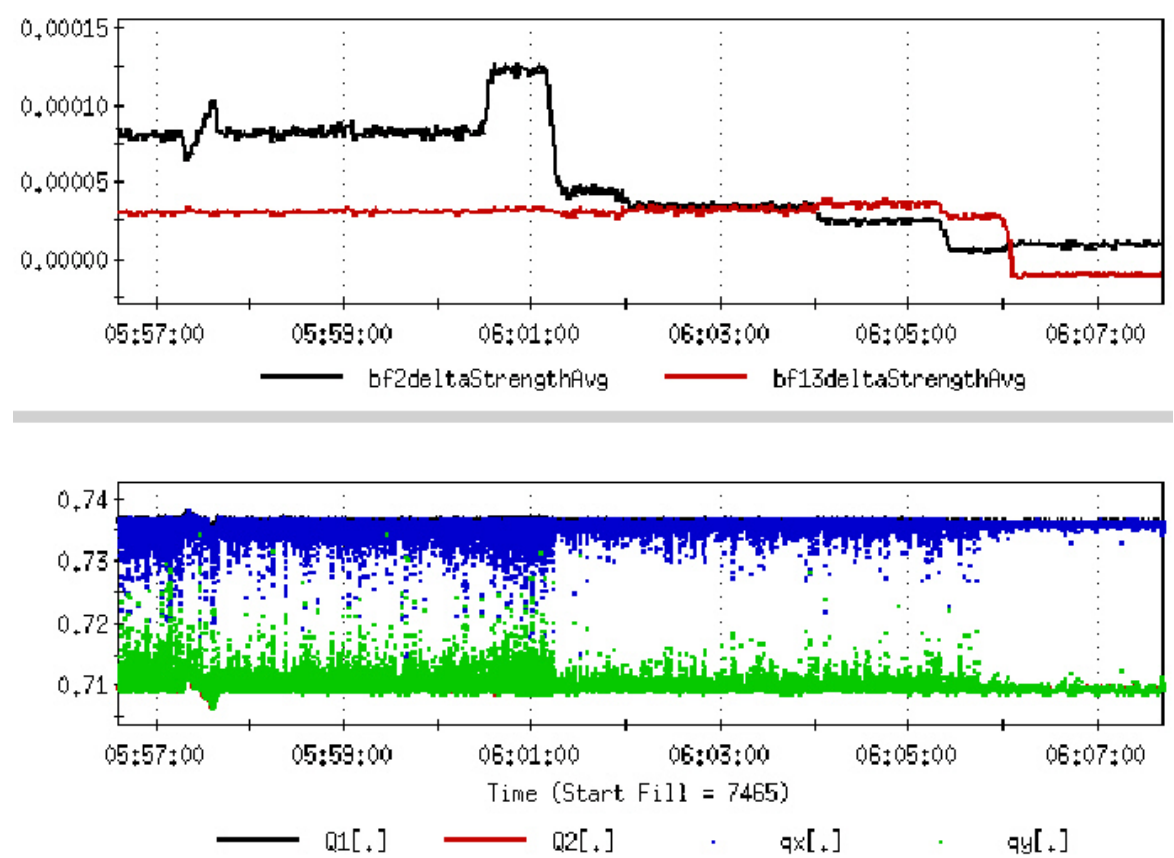

FIG. 10. (Color) Manual decoupling. 


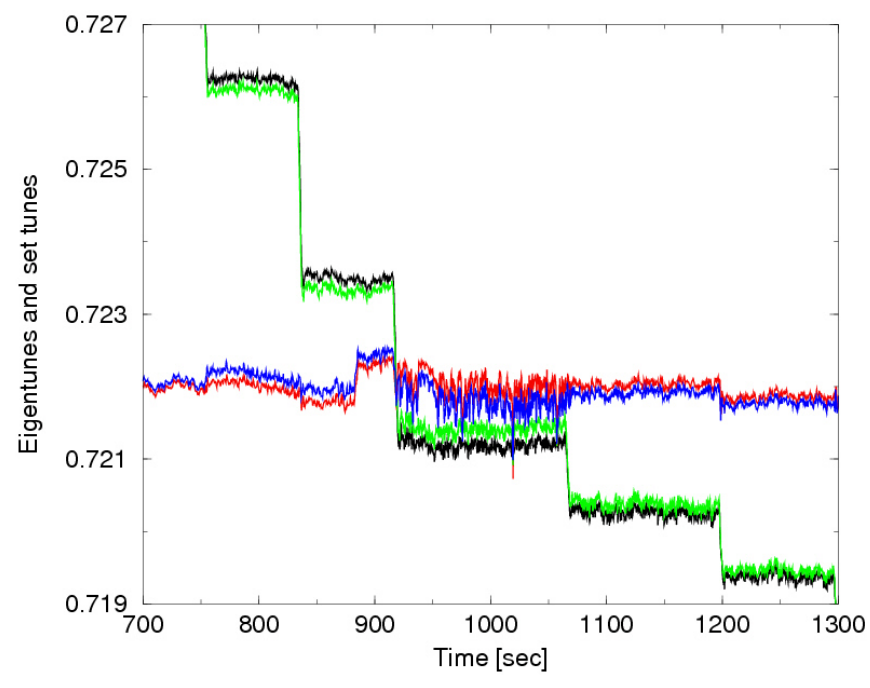

FIG. 11. (Color) Tune scan showing minimum tune split.

the coupling data. If tune feedback had been on in these figures, the feedback would have been driven unstable by the crossing of the set tunes.

Success of the tune feedback effort is predicated on decoupling. Therefore, the first objectives when beam became available in run 6 were to validate the coupling measurement, then close the loop on coupling feedback.

Figure 10 shows data taken early in run 6, during the week of machine setup in preparation for ramp development. The upper pane shows calculated correction strengths for the families F2 and F13, derived from the measured coupling. The lower pane shows set and measured tunes (measured tunes are obscured by the set tune data) during decoupling. It can be seen that as the requested skew correction strengths are adjusted towards zero, the set tunes converge on the measured tunes. In the leftmost portion of the figure, both the tunes and the requested family F2 correction strength can be seen to vary during a chromaticity measurement by the standard radial step technique. The variation in coupling indicated by the change in skew correction strength is a measure of skew chromaticity.

The commonly accepted fiducial measure of coupling is accomplished by moving the tunes as close as possible to determine the minimum tune split. This method is not often used because it perturbs machine operation, but is useful for comparing results with other measurement methods. From the eigenmode-based coupling measurement, skew strengths can be adjusted to zero the coupling, and set and measured tunes agree in this case. But the real proof that the tunes are decoupled is to measure the closest tune approach. Figure 11 shows data from a tune scan after the tunes were decoupled via the method shown in Fig. 10. It can be seen both that the set and measured tunes were in agreement, and that the closest tune approach was 0.001 or less.

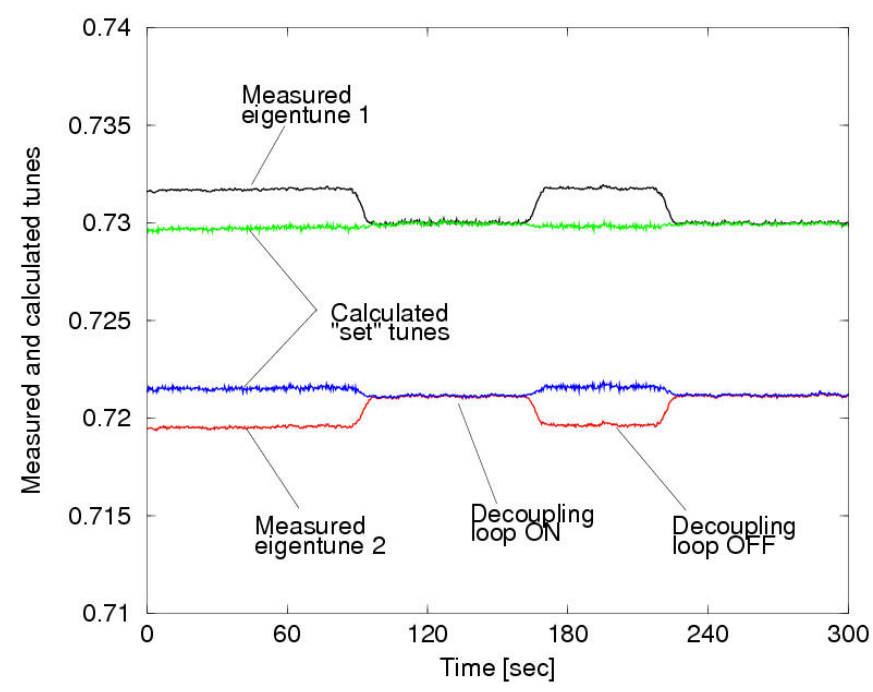

FIG. 12. (Color) Coupling feedback on and off.

Figure 12 shows set and measured tunes as the coupling feedback loop is turned on, then off, and then back on again. With the loop off the measured tunes are driven apart by the coupling. With the loop on the set and measured tunes are essentially the same.

Figure 13 shows data taken from 3 ramps during $100 \mathrm{GeV}$ ramp development. It should be noted that PLL loop gains must be adjusted manually during ramps with feedbacks on, to compensate the effect of chromaticity variations. This will be discussed in more detail in the following section. The uppermost pane in the figure shows set and measured tunes during the first acceleration ramp with feedbacks enabled. For the most part the correction from the coupling feedback was so good that the measured tune data is obscured by the set tune data. Loop gains in both the PLL tune tracker loop and the overall tune and coupling feedback loops were set somewhat high to insure tracking of fast changes. Additionally, PLL gains were manually adjusted through the ramp to accommodate changing beam conditions.

The middle pane shows the 11th and last of the $100 \mathrm{GeV}$ feedback ramps in blue. Magnet correction strengths were fed forward from ramp to ramp, so that by the end of this phase of ramp development the required corrections were small, and loop gains could be lowered. The improved quality of the data is clearly evident.

The lower pane shows data from the next ramp, with feedbacks off and corrections from the previous ramp fed forward. There were no deliberate orbit changes between the two ramps, and hence coupling was not changed significantly.

\section{AREAS FOR FURTHER INVESTIGATION}

During the course of this work two additional obstacles to making the system fully operational became clear. The first is the presence of large amplitude direct excitation of 

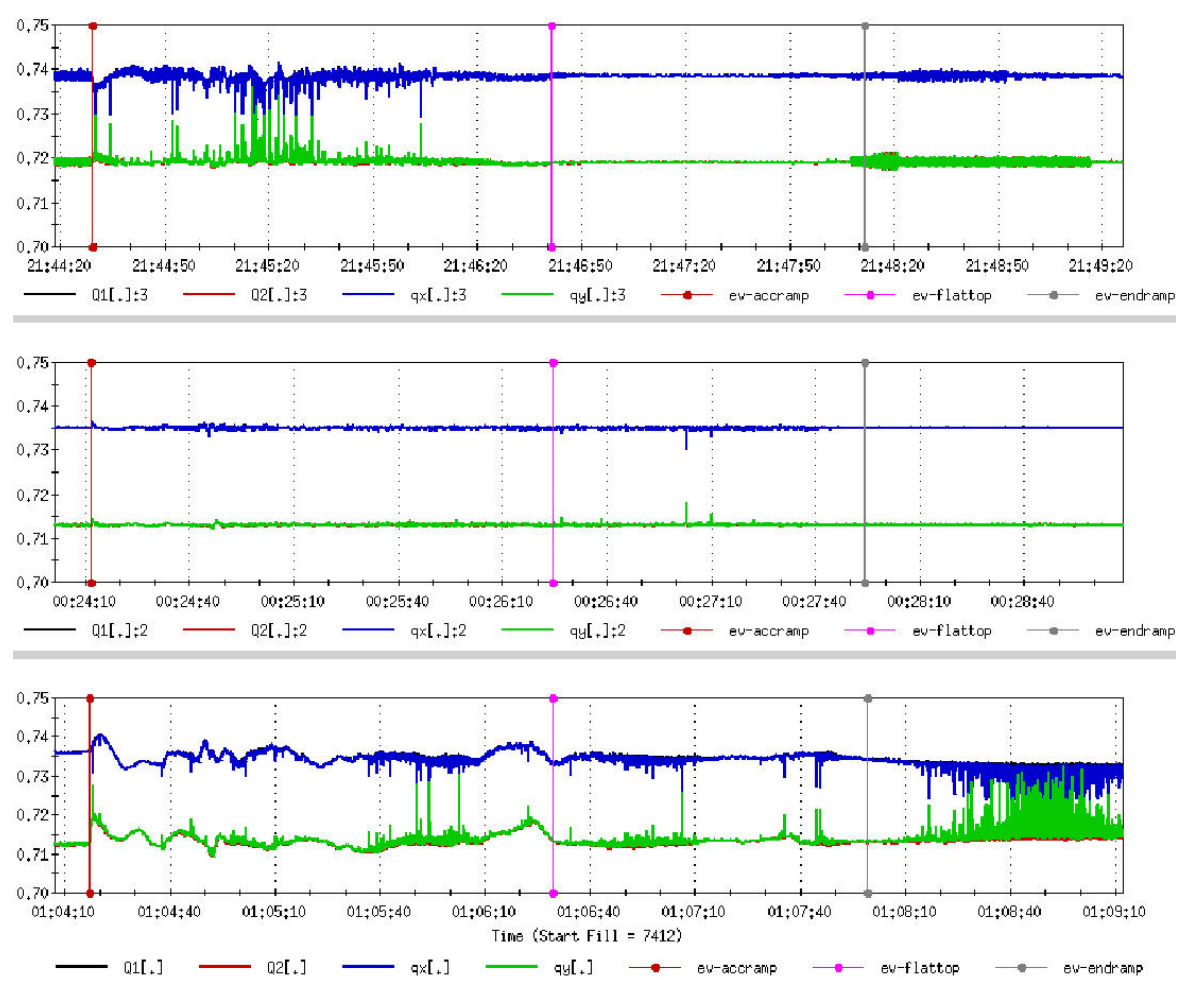

FIG. 13. (Color) Data from $100 \mathrm{GeV}$ development ramps in RHIC run 6.

the betatron resonances by high harmonics $(h \sim 350)$ of the $60 \mathrm{~Hz}$ power frequency, and the second is the need for precise chromaticity control (and possibly feedback) for proper operation of the tune tracker.

\section{A. Mains harmonics}

The 3D AFE delivers significant improvement in position measurement sensitivity, with estimated $10 \mathrm{~nm}$ resolution at RHIC. This AFE has been installed at the CERN PS and SPS, at the FNAL Tevatron, and at RHIC. Mains harmonics have been observed in the betatron spectrum at every installation [13]. Extensive studies have conclusively demonstrated that these harmonics result from direct excitation of the beam at the betatron frequency by high harmonics of the power line frequency. At RHIC the resulting spectral lines are as much as $40 \mathrm{~dB}$ above the 3D AFE noise floor at injection and store, and as much as $80 \mathrm{~dB}$ above the noise floor during ramping, when additional supplies are switched in to provide the field time derivative needed for ramping. The source of these harmonics is being actively investigated, and their possible origin is discussed in detail elsewhere $[13,14]$.

\section{B. Chromaticity}

For reliable tune tracking the overall loop gain of the PLL must be relatively constant. The beam-transferfunction portion of the loop gain is dependent on chromaticity. With large chromaticity both the amplitude and the phase slope of the betatron resonance response become small, and loop gain can be diminished to the extent that the PLL cannot track fast tune changes. Conversely, with small chromaticity the loop gain can become so large that the loop becomes unstable.

The pickup for previous generations of the RHIC tune feedback system utilized a stripline BPM resonated at $245 \mathrm{MHz}$, above the coherent spectrum. At that frequency the linewidth is dominated by broadening due to the high harmonic number, and studies showed that PLL performance was only weakly affected by chromaticity over the range from approximately 1-20 units. What effect was observed could be sufficiently compensated by feeding back on the kicker excitation to keep the amplitude response constant.

By virtue of its operating principle, the 3D AFE functions within the coherent spectrum, where the linewidth is dominated by chromaticity. In RHIC the $3 \mathrm{~dB}$ point of the coherent spectrum is at about $60 \mathrm{MHz}$ when the beam is bunched in the $28 \mathrm{MHz}$ acceleration cavities. Operational experience suggests that the tolerance of the 3D AFE PLL to chromaticity variations is less than that of the accelerator itself, and that chromaticity must be well controlled (in the range of 1 to 5 units) for reliable PLL operation. In addition to the expected effects on overall loop gain, the situation is aggravated by the fact that the 3D AFE functions as a peak detector, and is sensitive to the "microinstabilities" that precede the full blown head-tail instability caused by too small chromaticity. As chromaticity becomes small the 
PLL senses not the kicker excitation, but rather the selfexcitations of beam, and loses lock. As previously mentioned, this sensitivity to the effect of chromaticity on overall loop gain required that loop gains be manually adjusted during run 6 feedback ramps.

Past experience [15] with continuous chromaticity measurement using the PLL has been good. Data taken under automated sequencer control from three successive ramps during run 5 is shown in Fig. 14. This data was taken with the $245 \mathrm{MHz}$ resonant pickup, and the quality of the data near transition suffers due to excessive dynamic range as the bunch length shortens and extends up to the pickup frequency. The 3D AFE functions in the coherent spectrum, and does not have a problem with dynamic range around transition. The measurements were made with radial modulation of the beam with an amplitude of $200 \mu$ (this corresponds to a momentum deviation of $\sim 2 \times 10^{-4}$ ) at a rate of $1 \mathrm{~Hz}$. Excepting the time around transition, the measurement appears to be of adequate quality to permit feedback. It should also be mentioned that the algorithm for calculating chromaticity suffered from a sign ambiguity. This can be seen in the sign flips of the measured horizontal chromaticity at several places in the ramps. The source of this problem has been understood and corrected. Our expectation is that the quality of chromaticity measurement from the 3D AFE PLL will be similar to or better than that shown in the figure.

As a result of the observed sensitivity of the PLL to chromaticity, our plan for RHIC run 7 is to close the last loop, namely, chromaticity, in this multiloop feedback system. We intend to implement simultaneous tune, cou- pling, and chromaticity feedback as early in the run as is feasible.

\section{POSSIBLE IMPLICATIONS FOR LHC COMMISSIONING}

The out of the box success of simultaneous tune and coupling feedback in RHIC greatly improves the possibility that robust feedbacks can be implemented early in LHC commissioning. From the LHC perspective, the most urgent motivation for feedbacks is management of the chromatic effect of snapback at the beginning of the acceleration ramp [16]. The present understanding is that compensation of this effect will require real-time feedback control of chromaticity. This feedback is moving into the foreground of our efforts, motivated both by LHC needs for snapback control and the need to stabilize PLL loop gain in RHIC.

We emphasize that mains harmonics in RHIC are anomalously large, and their origin is not understood. This has many implications for the LHC. They impede needed system refinement at RHIC, and obstruct development work on chromaticity measurement and feedback. Their presence in the beam spectrum introduces spurious phase any time the tune is such that they are within the bandwidth of the PLL, an inevitable circumstance during the radial modulation chromaticity measurement with feedbacks off. They require careful selection of tunes when feedbacks are on. During normal operations with feedbacks off the effects of their spurious excitation diminish the confidence of nonspecialist users of the system, impeding its transition to
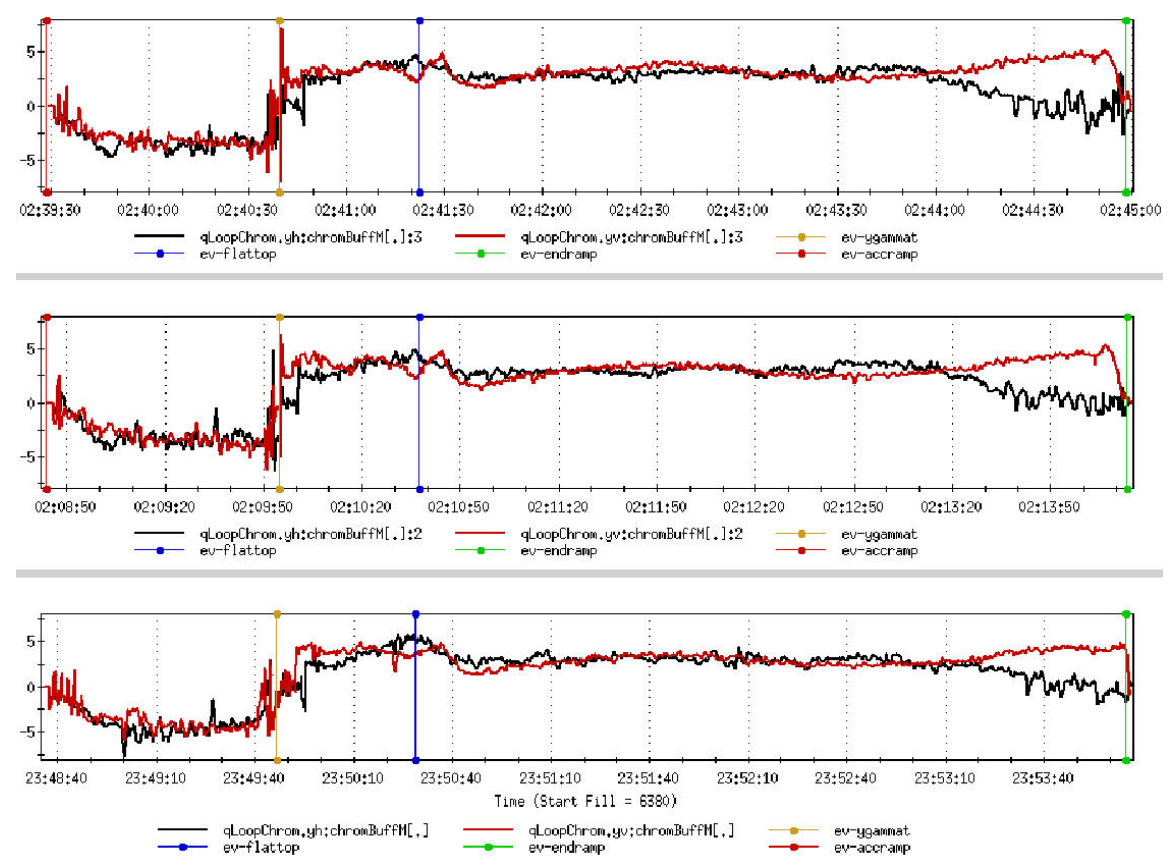

FIG. 14. (Color) Automated chromaticity measurement during three successive ramps. 
full operational status. Finally, until their origin is understood the possibility cannot be fully eliminated that a similar difficulty might arise in the LHC. Understanding and resolving this problem remains a high priority.

\section{ACKNOWLEDGMENTS}

The results presented here would not exist without the early foresight and guidance of Tom Shea, and the excellent foundation he provided to support these efforts. We gratefully acknowledge his many contributions. Hermann Schmickler initiated the collaborative effort between CERN and BNL. He, Mike Harrison, and Steve Peggs brought this collaboration into the U.S. LHC Accelerator Research Program (LARP), where it was expanded to include FNAL and has prospered under the oversight of Alex Ratti. The support of the LARP structure has been essential from a financial and organizational perspective, and additionally has enhanced the scientific and technical quality of the effort. The authors acknowledge support of the RHIC High Frequency Instrumentation Group in earlier efforts and the C-AD Beam Instrumentation Group in the present effort, and particularly Tom Russo, Tony Curcio, Ron Schroeder, and Al Weston. Finally, we acknowledge the tireless and efficient oversight of Vadim Ptitsyn in his role as run coordinator of RHIC run 6, as well as the steadfast long-term support and encouragement of Thomas Roser from the very beginnings of this effort. This work is supported by the U.S. DOE under Contract No. DE-AC02-98CH10886 and the U.S. LHC Accelerator Research Program (LARP).

[1] The tune feedback effort at RHIC was initially a BNL effort, was expanded into a bilateral collaboration with
CERN in 2002, and became a multilab LARP collaboration in 2003. For further information, see http://www. agsrhichome.bnl.gov/LARP/.

[2] P. Cameron et al., PAC 2003, Portland; http://accelconf. web.cern.ch/AccelConf/p03/PAPERS/ROAB009.PDF.

[3] P. Cameron et al., DIPAC 2005, Lyon; http://dipac2005. web.cern.ch/dipac2005/default.htm.

[4] R. Littauer, AIP Conf. Proc. 105, 921 (1982).

[5] M. Gasior and R. Jones, LHC-Project-Report853; http://doc.cern.ch/archive/electronic/cern/preprints/ lhc/lhc-project-report-853.pdf.

[6] M. Gasior and R. Jones, DIPAC 2005, Lyon; http:// dipac2005.web.cern.ch/dipac2005/default.htm.

[7] Y. Luo et al., PAC 2005, Knoxville; http://accelconf.web. cern.ch/AccelConf/p05/PAPERS/TPAP052.PDF. A much expanded version is available at http://www. agsrhichome.bnl.gov/AP/ap_notes/ap_note_174.pdf.

[8] Y. Luo et al., preceding Article, Phys. Rev. ST Accel. Beams 9, 124001 (2006).

[9] R. Jones et al., DIPAC 2005, Lyon; http://dipac2005.web. cern.ch/dipac2005/default.htm.

[10] G. Lambertson and D. Goldberg, A Primer on Pickups and Kickers (AIP, New York, 1990), p. 249.

[11] G. Decker, BIW 1994, Vancouver.

[12] C. Schultheiss et al., EPAC 2002, Paris; http://accelconf. web.cern.ch/AccelConf/e02/PAPERS/THPRI041.pdf.

[13] P. Cameron et al., DIPAC 2005. Lyon; http://dipac2005. web.cern.ch/dipac2005/default.htm.

[14] P. Cameron et al., C-AD AP note 253, 2006. This note is available at http://www.agsrhichome.bnl.gov/AP/ ap_notes/.

[15] S. Tepikian et al., EPAC 2002, Paris; http://accelconf. web.cern.ch/AccelConf/e02/PAPERS/THPRI075.pdf.

[16] S. Fartoukh and J. P. Koutchouk, Functional Specification LHC-B-ES-004， 2002; http://www.rhichome.bnl.gov/ LARP/papers/LHC_tune_spec.pdf. 